\title{
Pseudoluxación C2-C3 en la infancia
}

\section{Pseudoluxation C2-C3 in childhood}

\author{
Marina Angulo Tabernero ${ }^{1}$, Enrique Suñén Sánchez ${ }^{1}$, Diego Peña Jiménez ${ }^{1}$, Antonio Loste Ramos ${ }^{1}$, \\ Antonio Tabuenca Sánchez ${ }^{1}$, Alfredo Chárlez Marco ${ }^{1}$, Javier Rodríguez Vela ${ }^{1}$
}

\section{Resumen:}

Las lesiones traumáticas de la columna cervical son infrecuentes en los niños, representando el $1 \%$ de las fracturas pediátricas y el $2 \%$ de todas las lesiones raquídeas. Es importante conocer que la columna cervical en la infancia presenta variaciones anatómicas que en ocasiones pueden interpretarse como patológicas ya que en los niños una adecuada exploración física muchas veces puede ser complicada. El objetivo de este caso clínico es mostrar un hallazgo radiológico fisiológico de la columna cervical infantil que tiende a ser malinterpretado como patológico: la pseudosubluxación C2-C3.Se presenta un caso de un niño de 5 años de edad que acude a urgencias de traumatología pediátrica de nuestro centro por dolor cervical tras caída desde un tobogán. Se realizan radiografías cervicales de urgencias observando una disrupción de la línea cervical anterior y posterior entre C2 y C3. Una adecuada anamnesis junto con un meticuloso examen clínico y radiológico permite su correcto diagnostico sin necesidad de otras pruebas complementarias. La pseudoluxación anterior de C2 sobre C3 se puede estar presente en el $40 \%$ de los niños menores de 8 años. Este hallazgo puede diferenciarse de la luxación traumatic valorando la línea espinolaminar o línea de Swischuk, línea entre el borde anterior de las apófisis espinosas de C1 a C3. La interpretación adecuada de las radiografías de la columna cervical en la infancia requiere del conocimiento de estas variantes radiológicas y de las peculiares características de este segmento vertebral en la edad pediátrica.

Palabras clave: pseudosubluxacion C2-C3; infantil, listesis cervical; línea espinolaminal

\section{Abstract:}

Cervical spine injury is rare in children. It is seen primarily in those who sustain significant, severe blunt trauma, occurring in 1 to 2 percent of such cases. The interpretation of cervical spine radiographs and the clinical examination in children may be difficult.The objective of this case clinic is to report on a physiological radiographic finding of the cervical spine in childhood that trends to be misjudged as pathologic: C2-C3 pseudosubluxation. We presented a boy, aged 5 years, who came to our Traumatic pediatric emergency center for cervical pain after falling from a height. In lateral radiographs we observed a disruption of the alignment of the anterior and posterior vertebral body line in C2-C3 space. A detailed anamnesis as well as a meticulous radiological and clinical exploration, allow to establish the right diagnosis in most cases, without any other complementary probe. Pseudosubluxation of C2 anterior to C3 is seen in approximately 40 percent of children under the age of 8 years. Pseudosubluxation can be differentiated from true subluxation by evaluating the spinolaminal line (Swischuk), line between the anterior aspects of the $\mathrm{C} 1$ and $\mathrm{C} 3$ spinous processes. The suitable radiographic interpretation of the cervical spine in children requires the knowledge of the peculiarities of this segment in the pediatric age.

Keywords: C2-C3 pseudosubluxation; childhood; cervical listhesis; spinolaminal line. 


\section{Pseudoluxación C2-C3}

\section{Introducción}

Las lesiones traumáticas de la columna cervical en los niños son infrecuentes, representando únicamente el $1 \%$ de las fracturas pediátricas. Estas suelen estar asociadas a traumatismos de gran energía como accidentes de tráfico o precipitados desde altura ${ }^{1}$. La columna cervical inmadura presenta numerosas variantes anatómicas de la normalidad, hallazgos fisiológicos y radiológicos que pueden ser malinterpretados como patológicos. Esta diferencia puede ser especialmente dificultosa en el contexto de niños pequeños en los que la adecuada exploración clínica puede ser complicada.

\section{Caso Clínico}

Se presenta el caso clínico de un paciente varón de 5 años de que sufre traumatismo cervical al caerse de un tobogán de una hora de evolución.

Los padres acuden con el niño a las Urgencias Pediátricas de nuestro centro al objetivar discreta desviación hacia la derecha de la cabeza del niño asociado a rigidez y dolor a la movilización cervical en relación al traumatismo.

El examen físico del paciente se veía dificultado por su corta edad. La exploración neurológica de fue normal. A la exploración se objetivó la reticencia del niño a girar la cabeza para localizar objetos en el lado derecho y presentaba tortícolis con inclinación cefálica hacia el hombro izquierdo con discreta desviación del mentón hacia el lado derecho. A la palpación presentaba dolor a nivel de la musculatura cervical derecha.

En el estudio radiográfico inicial se objetivaba el desplazamiento anterior de varios milímetros del cuerpo vertebral de C2 sobre C3 (Figura 1). Sin embargo, un examen detallado de las radiografías evidenciaba que aunque existía una aparente disrupción de las líneas vertebral anterior y posterior, la línea espinolaminar (línea de Swischuk) estaba mantenida, de modo que las corticales anteriores de las apófisis espinosas de C1-C2-C3 están alineadas en un trazo sin disrupciones.
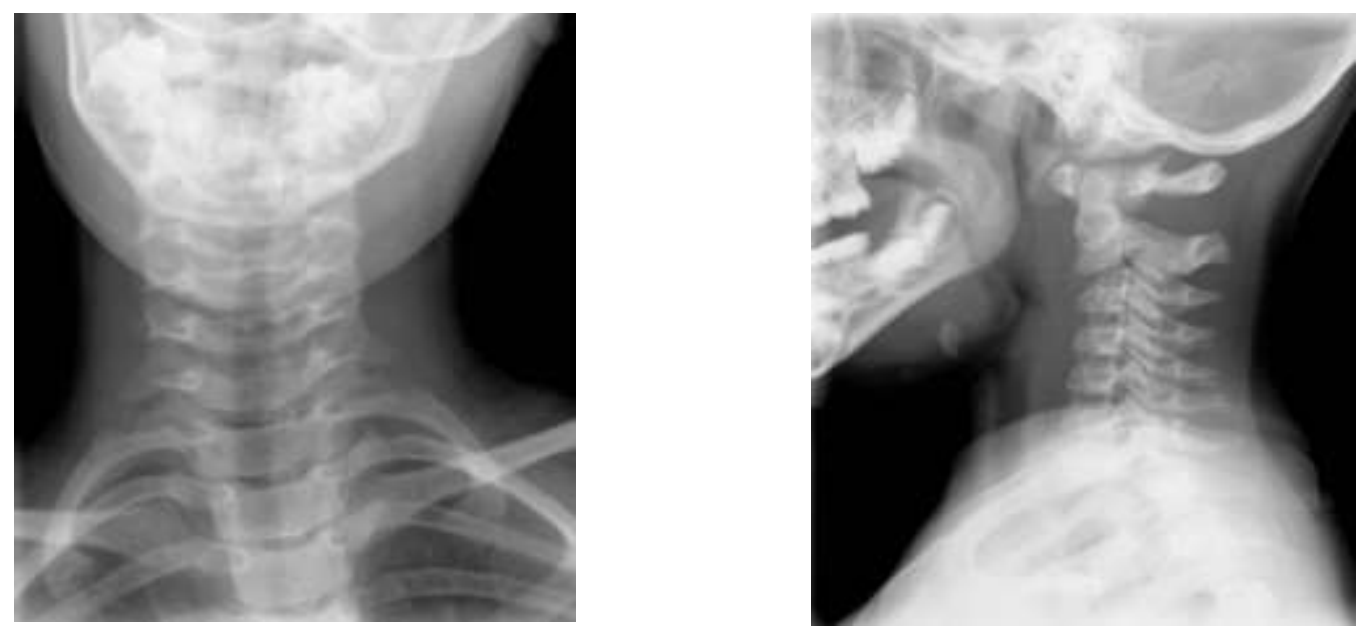

Figura 1: Proyección AP y lateral de la región cervical del paciente que muestra la desviación lateral del cuello y el desplazamiento anterior de varios milímetros del cuerpo vertebral de C2 sobre C3. 


\section{Pseudoluxación C2-C3}

Ante estos hallazgos radiológicos, el mecanismo lesional de baja energía y la exploración física anodina se decidió realizar una radiografía en extensión cervical en la que se observó la corrección del desplazamiento de C2 sobre C3 (Figura 2).

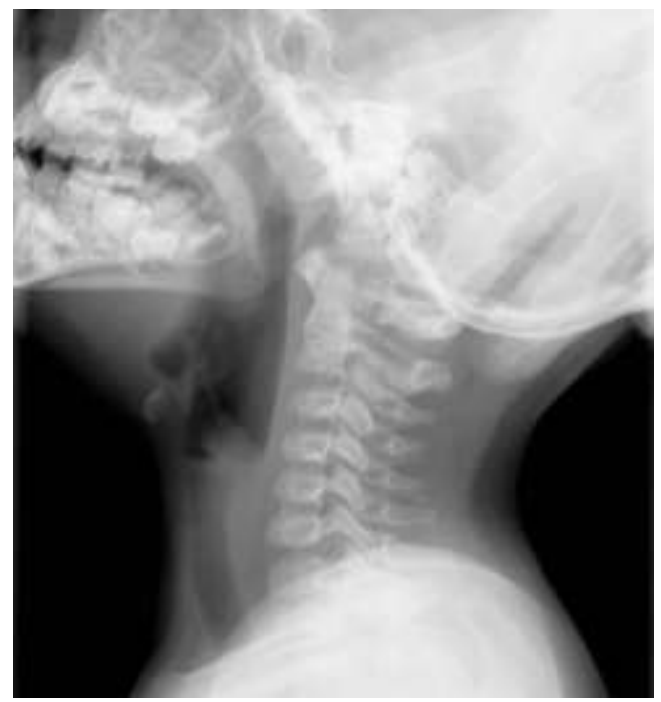

Figura 2: Proyección lateral del mismo paciente que muestra la corrección de la pseudoluxación C2-C3 en posición de extensión cervical.

El paciente fue diagnosticado de una contractura cervical y dado de alta del Servicio de Urgencias para control ambulatorio en la consulta de Traumatología Infantil de nuestro hospital en el plazo de 7-10 días con un collarín blando, recomendaciones de calor local e ibuprofeno como tratamiento de su contractura cervical.

La evaluación del niño fue satisfactoria. Se objetivó mejoría clínica con el tratamiento pautado a los pocos días y retomó su actividad diaria previa la revisión por Traumatología Infantil.

\section{Discusión}

La pseudosubluxación C2-C3 es la variante de la normalidad más frecuente en la infancia, pudiéndose encontrar hasta en el $46 \%$ de los niños menores de 8 años, seguida de la pseudosubluxación C3-C4 ${ }^{2}$.

En los niños se pueden ver estos desplazamientos, de hasta 4-5 mm de un cuerpo vertebral sobre otro, debido a la mayor elasticidad ligamentosa, el posicionamiento horizontal de las apófisis articulares por su menor desarrollo, el tono muscular disminuido y el tamaño relativo mas grande de la cabeza del niño pequeño. Habitualmente se pueden observar mas acentuados en actitud de flexión cervical, sin existir lesión osteoligamentaria asociada ${ }^{3}$.

Es fundamental realizar una detallada anamnesis, con especial atención al posible mecanismo lesional, y un riguroso examen clínico y radiológico. En las lesiones traumáticas graves de la columna cervical el paciente puede encontrarse con un nivel Glasgow bajo por asociarse a traumatismo craneal, permanecer en decúbito supino con resistencia a la movilización por el dolor o incluso sujetarse la cabeza con las manos debido a la sensación de inestabilidad. En el caso de la pseudosubluxación C2-C3, el 


\section{Pseudoluxación C2-C3}

paciente puede estar asintomático o referir molestias, en ocasiones intensas, causada por la contractura de la musculatura paravertebral cervical debido a un antecedente traumático menor ${ }^{4}$.

La evaluación radiográfica debe ser sistemática y completa, incluyendo las proyecciones anteroposterior y lateral de toda la columna cervical.

En la radiografía lateral debe valorarse ${ }^{1}$ (Figura 3):

- La alineación anterior y posterior de los cuerpos vertebrales.

- La distancia interespinosa: El aumento de la distancia entre las espinosas de algún nivel puede hacer sospechar una lesión del complejo ligamentoso posterior).

- El espacio prevertebral: El espacio prevertebral será normal en la pseudosubluxación mientras que se puede apreciar aumento de partes blandas en los casos de luxación y fractura-luxación en los que se produzca una rotura del ligamento común vertebral anterior, con el consiguiente sangrado.

- La línea espinolaminar o línea de Swischuk (línea trazada desde la cortical anterior de la apófisis espinosa de C1 hasta la cortical anterior de la apófisis espinosa de C3): En la pseudosubluxación C2-C3 la cortical anterior de la apófisis espinosa de C2 debe estar en continuidad con esta línea o una distancia menor de 1'5 - 2mm, mientras que en la verdadera subluxación la cortical anterior de la apófisis espinosa de C2 no se alinearía con este trazo en más de $2 \mathrm{~mm}$.
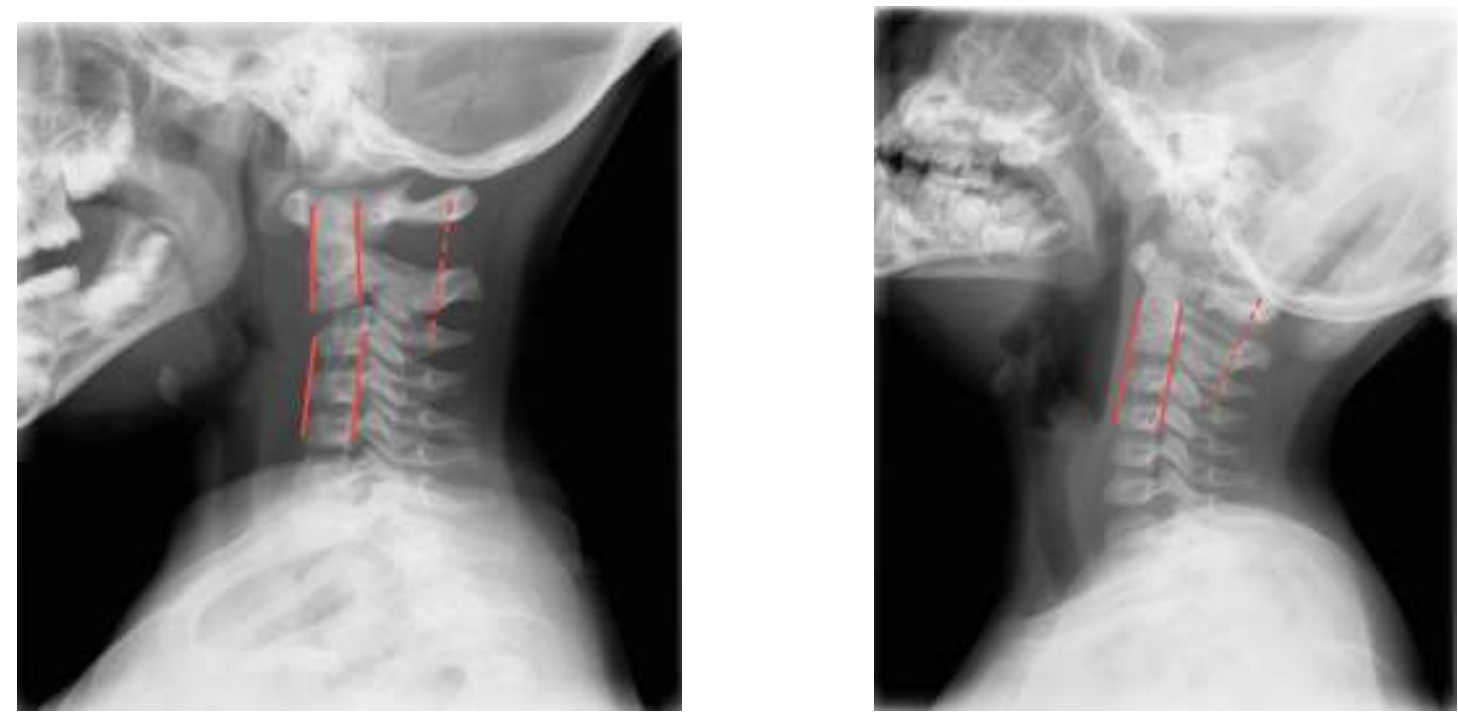

Figura 3: Proyección lateral y funcional en extensión que muestran la interrupción líneas anterior y posterior de los cuerpos vertebrales a nivel de C2-C3 en la proyección lateral y la correcta alineación en la proyección funcional en extensión (líneas continuas). Destacar el mantenimiento de la línea espinolaminar o línea de Swischuk en ambas proyecciones (línea discontinua).

Otra característica radiológica es que en la pseudosubluxación C2-C3 el desplazamiento entre el cuerpo vertebral de C2 y C3 es más marcado cuando las radiografías son tomadas en flexión cervical, corrigiéndose en posición neutra o en discreta extensión, mientras que la subluxación verdadera persistiría en cualquier posición ${ }^{1}$. Si persiste la 


\section{Pseudoluxación C2-C3}

duda pueden realizarse radiografías funcionales, siempre y cuando no exista déficit neurológico asociado, o bien completarse el estudio con una tomografía computarizada.

\section{Conclusiones}

El conocimiento de estas variantes, especialmente del segmento cervical superior, resulta de gran utilidad para establecer correctamente el diagnóstico, aplicar el tratamiento oportuno y evitar pruebas complementarias innecesarias, ya que son hallazgos fisiológicos que pueden ser interpretados erróneamente como patológicos.

\section{Bibliografía}

1. Lustrin ES, Karakas SP, Ortiz AO, Cinnamon J, Castillo M, Vaheesan K, et al. Pediatric Cervical Spine: Normal Anatomy, Variants, and Trauma. RadioGraphics. 2003;23(3):539-60.

2. Ogden JA. Radiology of postnatal skeletal development. Skeletal Radiol. 1984;12(3):169-77.

3. Wilkins R. Rockwood and Wilkins-Fractures in Children. 5th edition. Philadelphia: Lippincott Co.; 2003. 809-46p.

4. Ghanem I, El Hage S, Rachkidi R, Kharrat K, Dagher F, Kreichati G. Pediatric cervical spine instability. J Child Orthop. 2008;2(2):71-84. 